\title{
Diagnosis of Acute Pancreatitis by Computed Tomographic Evaluation
}

\author{
Banuprakash $\mathbf{S}^{1}$ \\ ${ }^{1}$ Associate Professor, Department of Radio-Diagnosis, Sri Siddhartha Medical College, Agalakote, B H Road, Tumkur- 572107, \\ India
}

Corresponding author: Dr Banuprakash S, Associate Professor, Radiologist, THS Lab, IMA Building, Town Hall Circle, Tumkur -572101, India

DOI: http://dx.doi.org/10.21276/ijcmsr.2019.4.1.26

How to cite this article: Banuprakash S. Diagnosis of acute pancreatitis by computed tomographic evaluation. International Journal of Contemporary Medicine Surgery and Radiology. 2019;4(1):A105-A107.

\section{A B S T R A C T}

Introduction: The size of the normal pancreas can be measured in several ways. The best antero-posterior measurements of the normal gland were made by Kreel and Sandin with CT, head: $23+3 \mathrm{~mm}$; neck: $19+2.5 \mathrm{~mm}$; body: $20+3 \mathrm{~mm}$; tail: 15 $+2.5 \mathrm{~mm}$. These measurements with CT differ from ultrasound measurements which are tend to be smaller. Study aimed at diagnosis of acute pancreatitis by computed tomographic evaluation.

Material and methods: A clinico-statistical cross sectional study was done at a tertiary care centre radiodiagnosis department, Tumkuru. The studied parameters of 30 cases included clinical suspicion/diagnosis of acute pancreatitis, deranged serum amylase and serum lipase levels going in favour diagnostic criteria of acute pancreatitis. 2

Results: Maximum number of patients are belongs to age group of 31-40 years ( 11 cases), followed by $41-50$ years ( 10 cases) and majority of cases are male (24 cases). Diffuse/focal pancreatic enlargements were seen in 22 cases followed by Peripancreatic fat stranding in 18 cases and peri-pancreatic fluid in 10 cases.

Conclusion: Acute The severity of inflammatory process with staging, detection of pancreatic necrosis, depiction of local complications along with grading of severity of acute pancreatitis can be accurately detected by contrast enhanced computed tomography which serves as a important diagnostic tool .

Keywords: Acute Pancreatitis, CT Scan, Contrast

\section{INTRODUCTION}

The size of the normal pancreas can be measured in several ways. The best antero-posterior measurements of the normal gland were made by Kreel and Sandin with CT, head: 23 +3mm; neck: $19+2.5 \mathrm{~mm}$; body: $20+3 \mathrm{~mm}$; tail: $15+$ $2.5 \mathrm{~mm}$. These measurements with CT differ from ultrasound measurements which are tend to be smaller. The pancreatic duct diameter is about $2-4 \mathrm{~mm}$ and that of the intrapancreatic common bile duct is usually less than $6-7 \mathrm{~mm}$. The density of the non-enhanced pancreas is between 30 50 Hounsfield units. It increases to100-150 HU after the intravenous administration of iodine based contrast agents. Rapid acquisition of images after a bolus injection allows visualization of normal enhancement during parenchymal and portal venous phases. ${ }^{1,2}$

Atlanta Symposium ${ }^{1}$ defines, acute pancreatitis as an acute inflammatory process of the pancreas that may also involve peri-pancreatic tissues and/or organ systems. 4

The Patho-Physiology of acute pancreatitis is generally considered in three phases.

1. In the $1^{\text {st }}$ phase, premature activation of trypsin within pancreatic acinar cells leads to activation of a variety of injurious pancreatic digestive enzymes. 5

2. In the second phase, there is intra-pancreatic inflammation.

3. In the third phase, there is extra-pancreatic inflammation. Computed tomography with contrast is the choice for the diagnosis and grading of AP17, 18. A three-phase protocol (non-enhanced phase, pancreatic parenchymal phase 20-40 seconds and portal venous phase 55-75seconds) can be used for the initial assessment of acute pancreatitis. Positive oral contrast may be administered after the control scan. Nonenhanced phase is useful in detecting calcification/ calculi. So the initial non-enhanced study is done. The normal pancreatic tissue shows greatest enhancement during parenchyma phase and hence this is taken as the optimal phase for assessment of pancreatic necrosis. Serial imaging with CT is usually done using a single-phase technique in the portal venous phase.

Sometimes, the presumed danger that IV administration of iodinated contrast material can aggravate the severity and duration of acute pancreatitis has put a caveat regarding IV contrast usage. However the current opinion is that the benefits of IV contrast administration outweighs the potential risks. . ${ }^{3,4}$

The pancreas may enhance uniformly in cases of mild acute pancreatitis and may be termed normal or enlarged with a variable amount of increased attenuation in the adjacent fat, which may be synonymous with the terminology "stranding". Non enhancement of all or part of the gland is termed 
"necrosis". CT is highly specific denoting necrosis when greater than one-third of the gland is involved and is non enhancing non-enhancing 14 . In acute pancreatitis, necrosis manifests between one to two days after the onset of initial inflammation. Hence, CT done with in $1^{\text {st }} 12$ hours may be falsely positive and re assuring. Acute pancreatitis may usually progress to pancreatic abscess formation seen with in 4 to 6 weeks after the initial onset. CT findings in such cases are seen as an area of low attenuation containing pus and a thick wall that may enhance after giving IV contrast Air bubbles may be found within the collection. The most important imaging markers of acute pancreatitis having prognostic relevance are necrosis and abscess which may need intervention by interventional radiologist or surgeons. 5,6

Study aimed at diagnosis of acute pancreatitis by computed tomographic evaluation.

\section{MATERIAL AND METHODS}

Cross-sectional study was conducted in Radio-diagnosis department of tertiary care hospital, Tumkuru. The studied parameters of 30 cases included clinical suspicion/diagnosis of acute pancreatitis, deranged serum amylase and serum lipase levels going in favour diagnostic criteria of acute pancreatitis

\section{Inclusion Criteria}

- Patients with clinical or diagnostic suspicion of acute pancreatitis with focus on serum amylase and serum lipase levels.

\section{Exclusion Criteria}

- Pancreatic tumor.

- Pancreatic trauma.

Equipment: Toshiba Asteion single slice spiral Computed Tomography scan.

Protocol: The abdomen and pelvis were taken in a Plain and post-contrast series .Abdomen and pelvis with a thickness of $5 \mathrm{~mm}$ and $3 \mathrm{~mm}$ respectively were taken in a contiguous axial sections and region of interest in the cranio-caudal direction from the level of the xiphisternum to pubic-symphysis, before and after administration of oral and IV iodinated contrast of $80-100 \mathrm{ml}$.

\section{RESULTS}

Maximum number of patients are belongs to age group of 31-40 years (11 cases), followed by $41-50$ years (10 cases) and majority of cases are male (24 cases).

Diffuse/focal pancreatic enlargements were seen in 22 cases followed by Peri-pancreatic fat stranding in 18 cases and

\begin{tabular}{|l|c|c|c|}
\hline Age group & Male & Female & Total \\
\hline$<30$ Years & 01 & 00 & 01 \\
\hline 31-40 Years & 09 & 02 & 11 \\
\hline 41-50 Years & 07 & 03 & 10 \\
\hline 61-70 Years & 06 & 01 & 07 \\
\hline$>70$ Years & 01 & 00 & 01 \\
\hline Total & 24 & 06 & 30 \\
\hline \multicolumn{4}{|l|}{ Table-1: Age and sex wise distribution of cases avn cases } \\
\hline
\end{tabular}

\begin{tabular}{|l|c|c|}
\hline \multirow{2}{*}{ CT findings } & \multicolumn{2}{|c|}{ Number of patients } \\
\cline { 2 - 3 } & Present & Absent \\
\hline Peri-pancreatic fat stranding & 18 & 12 \\
\hline Diffuse/focal pancreatic enlargement & 22 & 08 \\
\hline Peri-pancreatic fluid collection & 10 & 20 \\
\hline \multicolumn{2}{|c|}{ Table-2: Distribution of cases based on ct findings } \\
\hline
\end{tabular}

peri-pancreatic fluid in 10 cases.

\section{DISCUSSION}

Maximum number of patients belonged to age group of 31-40 years ( 11 cases), followed by $41-50$ years (10 cases) and majority of cases were male ( 24 cases). 22 cases showed diffuse or focal pancreatic enlargement. Peri-pancreatic fat stranding was seen in 18 cases followed by peri-pancreatic fluid in 10 cases. Acute pancreatitis was a commonly dangerous condition with a widely variable clinical course . The imaging modalities like CT and the radiologist place a vital role in management of such patients, including diagnosis, staging, treatment of complication, as well as identifying the underlying etiology. The objective of this article was (i) to familiarise the reader with the pathophysiology of acute pancreatitis, the appearances of the various stages of pancreatitis, the evidence for the use of staging classifications and the associated complications and (ii) to review current thoughts on optimising therapy.

Out of 102 patients suffering with acute pancreatitis diagnosed with abdominal CT scan, nearly 28 attacks were clinically severe and 72 clinically mild at one week and at 6 weeks of admission. Ninety three admission scans, 85 one week scans, and 52 six week scans were abnormal. The cause of the pancreatitis could be obtained from 28 (27\%) of admission scans, with a findings of fatty liver in CT(Sensitivity of $21 \%$ and specificity of $100 \%$ ) for alcoholic aetiology. The gall stone aetiology with (sensitivity of $34 \%$ and specificity of $100 \%)$. The pancreatic size indices of those patients with severe attacks were significantly greater than those with mild attacks on admissions, at 1 week and at 6 weeks ( $p$ less than 0.004). CT had detected 14 pseudocysts, among them 5, 36\% are clinically apparent. The pseudocyst size indices (max anteroposterior $x$ max transverse measurement) of the pseudocysts which were clinically apparent were significantly greater than those which were not apparent ( $p$ less than 0.01 ) and only those pseudocysts with a size index greater than or equal to $15 \mathrm{~cm} 2$ required treatment. ${ }^{8}$

A prospective study conducted in rural hospital from October 2011 to August 2013 in M.V. Jayaraman Medical College, sixty cases diagnosed as acute pancreatitis were included in this study. CECT of abdomen and pelvis were done on these patients, which were graded according to the modified CT severity index. The mean age of patients in the study was $37.18+11.45$ years. The maximum patients were in the age group of 25 to 35 years (36.6\%). The next group with maximum patients was in the 36 to 45 years group (26.6\%). The minimum age of patients was 17 years and maximum age was 62 years with a minimum number of patients seen below the age of 20 years. Most of the patients were male (85\%) as compared to female (15\%). No association of age and gender 
was noted with severity of pancreatitis in our study. These observations was similar to that of a study conducted by Lankish et al 52 on 602 patients of acute pancreatitis which showed no correlation between age, gender with severity of acute pancreatitis. The study highlighted the maximum incidence of acute pancreatitis in age group 31 to 40 years similar to our study.

Alcohol was the most common cause of AP seen in 52 (86.6\%) patients, 6 (10\%) patients were having GB/CBD calculi and 3 (5\%) patients were having hyperlipidemia. Out of these one patient had both alcohol and CBD calculus. A study by Wongnai et al conducted in 90 patients showed $60 \%$ patient of alcohol, $18 \%$ patient of CBD/MPD calculi. ${ }^{9}$

The studies by Bollen et al and Mortele et al have classified grade 2 as mild, grade 4 and 6 as moderate and grade 8 and 10 as severe. The prognosis of patients with grade 2 and 4 pancreatitis was similar and milder than patients who had a grade of 6 as observed in our study, hence were grouped together in our study. The morphologic severity of pancreatitis was graded as mild in 86 (44\%), moderate in 75 (38\%), and severe in $35(18 \%)$ cases. The study had patients with severe pancreatitis as the minimum number of patients which is similar to our study. Most patients are of mild grade in our study that possibly explains early use of CECT usefulness in mild cases of AP. According to Bollen et al the MCTSI accurately correlated with extra-pancreatic complication that needed an intervention compared with clinical score indices (APACHE II). CT is the modality of choice for detecting the local complications. Thus the MCTSI is as 84 useful in predicting the severity of $\mathrm{AP}$ in terms of organ failure, detecting the local complications and confirming necrosis in AP. ${ }^{10,11}$

\section{CONCLUSION}

Contrast enhanced Computed Tomography as evolved as an important and vital diagnostic tool to access the severity of inflammation, detect necrotic pancreatic tissue along with local complication and grading of severity of the disease. The modified Mortele index scores depicted a stronger correlation for all outcome parameters in all the patients comparatively better than the Balthazar index. Revised Atlanta classification is more accurate than modified Mortele index and Balthazar severity index for assessing patient mortality and organ failure.

\section{REFERENCES}

1. Banks PA. Practice guidelines in acute pancreatitis. Am J Gastroenterol. 1996; 92(1):377-386.

2. Haaga JR, Dogra V, Forsting M, Gilkeson R, Kwon Ha H, Sundaram M. CT and MR imaging of the whole body. 5th ed. Philadelphia: Elsevier; 2003.p.1599-1667.

3. Uhl W, Roggo A, Kirschstein T. Influence of contrastenhanced computedtomography on course and outcome in patients with acute pancreatitis. Pancreas. 2002;24(3):191-197.

4. McMenamin DA, Gates LK. A retrospective analysis of the effect of contrastenhanced CT on the outcome of acute pancreatitis. Am J Gastroenterol.1996;91(5):1384-1387.

5. Brown A, Baillargeon JD, Hughes MD. Can fluid resuscitation prevent pancreatic necrosis in severe acute pancreatitis? Pancreatology. 2002;2(2):104-7.

6. Kalfarentzos F, Kehagias J, Mead N. Enteral nutrition is superior to parenteral nutrition in severe acute pancreatitis: Results of a randomized prospective trial. Br JSurg. 1997;84(1):1665-

7. Koo BC, Chinogureyi A, Shaw AS. Imaging acute pancreatitis. Br J Radiol. 2010; 83(986): 104-112.

8. London NJ, Neoptolemos JP, Lavelle J, Bailey I, James D. Serial computed tomography scanning in acute pancreatitis: a prospective study. Gut. 1989; 30(3): $397-$ 403.

9. Amogh VN. Computed tomographic evaluation of acute pancreatitis. Bengaluru. RGUHS. 2014.

10. Mortele K, Wiesner W, Intriere L, Shankar S, Kelly H. A Modified CT Severity Index for Evaluating Acute Pancreatitis: Improved Correlation with Patient Outcome. AJR. 2004;183(5):1261-1265.

11. Bollen T, Singh V, Maurer R. Comparative Evaluation of the Modified CT Severity Index and CT Severity Index in Assessing Severity of Acute Pancreatitis. AJR. 2011;197(3):386-392.

\section{Source of Support: Nil; Conflict of Interest: None}

Submitted: 10-01-2019; Accepted: 09-02-2019; Published online: 20-03-2019 